\title{
Translating Neurobiology to the Treatment of Dual Diagnosis: The Example of Nicotinic Receptors and Neurocognitive Endophenotypes in Schizophrenia
}

\author{
Alanna C. Bridgman • Kristen M. Mackowick • \\ Michelle S. Goodman • Rachel A. Rabin • Mera S. Barr • \\ Tony P. George
}

Published online: 25 September 2014

(C) Springer International Publishing AG 2014

\begin{abstract}
Endophenotypes are heritable traits associated with psychiatric illness. Certain endophenotypes associated with schizophrenia such as working memory and sensory gating may be linked to the high tobacco smoking prevalence in patients with schizophrenia. Evidence suggests a critical role for the nicotinic acetylcholine receptor system (nAChR) in the underlying pathophysiology of schizophrenia. Despite the negative consequences associated with tobacco use, patients with schizophrenia have a much more difficult time quitting smoking and remaining abstinent compared with their nonpsychiatric counterparts. Deficits in nAChR function may contribute to the cognitive deficits that are seen with the illness, such as deficits in working memory and sensory gating, and to co-morbid tobacco use disorders. Accordingly, treatments for tobacco use disorder in this
\end{abstract}

\section{T. P. George ( $\square)$}

Schizophrenia Division, Centre for Addiction and Mental Health (CAMH), 1001 Queen Street West, Unit 2 Room 118A, Toronto, ON, Canada M6J $1 \mathrm{H} 4$

e-mail: tony.george@camh.ca

\author{
A. C. Bridgman • K. M. Mackowick • M. S. Goodman • \\ R. A. Rabin · M. S. Barr • T. P. George \\ Division of Brain and Therapeutics, Department of Psychiatry, \\ University of Toronto, 250 College Street, Toronto, ON, \\ Canada M5T 1R8 \\ A. C. Bridgman \\ e-mail: Alanna.Bridgman@camh.ca \\ K. M. Mackowick \\ e-mail: Kristen.Mackowick@camh.ca \\ M. S. Goodman \\ e-mail: Michelle.Goodman@camh.ca \\ R. A. Rabin \\ e-mail: Rachel.Rabin@camh.ca \\ M. S. Barr \\ e-mail: Mera.Barr@camh.ca
}

population should focus on remediation of the diseaserelated endophenotypes that restore neurocognitive function and reduce the risk of smoking relapse.

Keywords Schizophrenia · Endophenotypes · Tobacco · Neurocognition $\cdot$ Nicotine

\section{Introduction}

Approximately $60-80 \%$ of patients with schizophrenia smoke tobacco [1], compared with $20 \%$ of the general population [2]. While these data indicate an increased vulnerability to nicotine use among patients with schizophrenia, the direction of the relationship between tobacco use and schizophrenia is still unclear. While it may be true that patients with schizophrenia use nicotine to remediate symptoms of their illness and medication side effects, the more likely possibility is that deficits in the nicotinic acetylcholine receptor system (nAChR) increase an individual's vulnerability to tobacco use $[3,4]$.

Cognitive deficits are a core feature of schizophrenia and are linked to the nAChR system [5-7]. Neurophysiological disturbances, such as deficits in sensorimotor gating or cortical inhibition, are thought to underlie these cognitive impairments, thereby constituting putative endophenotypes associated with the disorder. Accordingly, dual diagnosis treatment should target these endophenotypes to potentially remedy these cognitive deficits and nicotine dependence in this population.

In this review, we will discuss the neurobiology of nicotine addiction as it relates to schizophrenia, as well as the effects of nicotine on cognitive functioning in this population. Furthermore, neurocognitive endophenotypes and their relationship to nicotine receptor dysfunction will be explored. Finally, we will evaluate the efficacy of putative nAChR-targeted 
treatments toward alleviating these cognitive deficits. Future directions for research in the area of schizophrenia and tobacco use disorder will be considered, with a focus on directing the classification and treatment of nAChR-mediated endophenotypes in schizophrenia.

\section{The Neurobiology of Tobacco Addiction in Schizophrenia}

Converging lines of research have focused on the shared vulnerability between nicotine use and schizophrenia, emphasizing the putative dysregulation of $\mathrm{AAChR}$ systems in schizophrenia $[8,9]$. Nicotine is the main psychoactive ingredient in tobacco smoke. When a cigarette is smoked, nicotine binds to the $\alpha 4 \beta 2 \mathrm{nAChR}$, the primary site of action of nicotine. These receptors are also activated by the endogenous neurotransmitter acetylcholine. The nAChRs situated on mesolimbic dopamine neurons that project from the ventral tegmental area (VTA) to the nucleus accumbens (NAc) are of utmost importance when studying nicotine addiction. Activation of nAChRs on mesolimbic dopamine neurons leads to dopamine release in the NAc, which helps to facilitate the addictive processes involved in chronic tobacco use.

Due to the high prevalence of cigarette smoking in schizophrenia, nAChRs are a prime intervention target in the pathophysiology of tobacco use disorder comorbidity in schizophrenia. The idea that common mechanisms may underlie both tobacco use disorder and schizophrenia was supported by a recent study examining the relationship between the genetics of nicotine use disorder and familial risk for schizophrenia. The results indicate that levels of smoking were much higher in first-degree relatives of schizophrenia patients than in a comparison group [10]. Furthermore, nAChRs may also play a role in mediating the cognitive deficits associated with schizophrenia (see Section "Nicotinic Systems and Neurocognitive Endophenotypes in Schizophrenia"), perhaps suggesting a vulnerability to both nicotine dependence and the manifestation of cognitive deficits in schizophrenia.

\section{Nicotinic Systems and Neurocognitive Endophenotypes in Schizophrenia}

The acute pharmacological effects of nicotine have been proposed to enhance cognitive function in patients with schizophrenia. Administering acute doses of nicotine to schizophrenic non-smokers can help tease apart the contributions of nicotine to cognitive functioning without the confounding effects of withdrawal-related symptoms or chronic upregulation of nAChRs. In non-smokers, transdermal nicotine (14 $\mathrm{mg}$ ) was found to increase reaction time and decrease omission errors on the Continuous Performance Task (CPT), though more significantly in schizophrenia [11], suggesting a more pronounced role of nicotine on attentional processes in schizophrenia relative to control non-smokers. Additionally, nicotine has been shown to improve visuospatial attention and smooth pursuit eye movements (SPEM) in both nonsmokers and smokers with schizophrenia, with no effect in nonpsychiatric groups [12]. These data suggest that acute nicotine has more of a pro-cognitive effect in patients with schizophrenia compared with non-psychiatric controls regardless of smoking status, potentially due to acute upregulation of nAChRs. The pro-cognitive effects of nicotine can be explored using neurocognitive endophenotypes present in schizophrenia that may reflect $\mathrm{nAChR}$ dysregulation.

Endophenotypes are stable and heritable indicators of disease states that cannot be identified with a simple biological test [13], and represent vulnerability to the disorder, rather than a diagnostic test of the disorder in question. There are some prerequisites that a potential endophenotype must meet in order to be useful in psychiatry: i) heritability and cosegregation with first-degree relatives, ii) association with a candidate gene region, and iii) state-independent consistency, whereby the deficit is present whether or not the illness is symptomatically active [14]. Since schizophrenia is a multigenetic and complex disorder, endophenotypes that segregate to a smaller number of genes are attractive treatment targets.

Deficits in working memory and sensory gating are examples of endophenotypes that are inherent to a range of psychiatric disorders, with the upstream genotype potentially involving abnormalities in $\mathrm{nAChR}$ function. Outlining these endophenotypes and their severity as it relates to schizophrenia may help to determine effective treatment approaches. Several nicotinic receptor-related endophenotypes have been suggested, including SPEM, pre-pulse inhibition (PPI), P50 auditory gating, and working memory. However, the question remains as to whether these endophenotypes confer a vulnerability to tobacco smoking in schizophrenia, or whether these endophenotypes share a common, underlying mechanism with nicotine dependence that could account for this significant comorbidity.

\section{Smooth Pursuit Eye Movements (SPEM)}

SPEM are a neurophysiological index of motion perception and processing, and have been consistently found to be deficient in both patients with schizophrenia and their first-degree relatives [14]. SPEM are relatively slow movements of the eye (usually less than 100 degrees per second). In contrast to saccadic movements, which aim to bring an object onto the fovea quickly, SPEM consists of a pursuit and a 'catch-up' phase, which acts to keep the object on the fovea throughout movement [15].

During SPEM tasks, patients with schizophrenia tend to make more intrusive saccadic movements than nonpsychiatric controls, potentially reflecting deficits in 
predictive and motion processing [15]. A functional magnetic resonance imaging (fMRI) study evaluating brain activation during a simple SPEM task showed greater activation of the posterior hippocampi and fusiform gyrus during the task, along with decreased activation in the frontal eye fields and occipital cortex [16]. These results suggest a potential inhibitory deficit of the hippocampus in schizophrenia patients, which is consistent with post-mortem evidence showing decreased numbers of hippocampal nicotinic receptors in patients with schizophrenia [17].

Although it is possible that patients with schizophrenia smoke to normalize deficits of eye movements and visual processing, it is more likely that nAChR deficits confer vulnerability to both deficits in SPEM and to nicotine use in these patients. Smokers with schizophrenia and control smokers were tested on a standard SPEM task after overnight abstinence and immediately after smoking a cigarette. Leading saccades were found to be significantly reduced in smokers with schizophrenia compared with the controls [18]. In a study of both schizophrenia and healthy control non-smokers and smokers, acute administration of nicotine significantly improved eye acceleration during smooth pursuit initiation in patients but not controls, but the initiation of eye acceleration was greater in patient smokers compared with patient nonsmokers [12]. These results suggest that nicotine may selectively enhance SPEM deficits in smokers with schizophrenia compared with nonsmokers.

\section{P50 Auditory Gating}

Sensory gating refers to pre-attentional habituation responses to the same auditory stimulus [19]. The ability to gate a response to the same stimulus is a necessary tool - without the ability to filter sensory information, the senses would be overwhelmed with irrelevant information. This may be the case for patients with schizophrenia, who consistently show deficits in P50 auditory gating. P50 refers to an event-related potential (ERP) that is used to assess sensory gating via electroencephalography (EEG) [19].

P50 gating deficits have been shown to be normalized in schizophrenia smokers compared with non-smoker controls. For example, a recent study in drug-naïve first episode schizophrenia patients showed that the schizophrenia group exhibited much worse P50 gating compared with non-psychiatric controls, yet schizophrenia smokers had significantly better P50 auditory gating compared with non-smoker schizophrenia patients [20]. A limitation to this study is that individuals were assessed 1 hour after smoking, and thus withdrawal effects were a confounding variable. Another study found similar results, such that drug-naïve smokers with schizophrenia display enhanced P50 compared with non-smokers with schizophrenia [21]. Many studies have also evaluated the effects of acute administration of nicotine on P50 sensory gating among patients and first-degree relatives [22-24]. In the relatives of patients with schizophrenia, nicotine administration normalizes deficient P50 gating [22], further implicating a pathophysiological role of nAChR in sensory gating in schizophrenia.

\section{Prepulse Inhibition}

Prepulse inhibition (PPI) is a neurophysiological phenomenon whereby a weak auditory stimulus $30-300 \mathrm{~ms}$ precedes stronger stimulus and reduces the elicited startle response. PPI is thought to reflect the ability of the brain to gate incoming repeated sensory information [25]. In patients with schizophrenia, deficits in inhibition of the startle response have been consistently replicated $[26,27]$. Of note, PPI deficits are also consistently found in the first-degree relatives of patients with schizophrenia, and thus are thought to be a relatively strong endophenotype candidate [27].

PPI may also be modulated by smoking status, such that smokers with schizophrenia display levels of PPI similar to control smokers compared with schizophrenia non-smokers [28•]. This study looked at four groups of participants: smokers with and without schizophrenia, and non-smokers with and without schizophrenia. A significant diagnosis $x$ smoking status $\times$ PPI interval interaction was found, such that non-smokers with schizophrenia showed deficits in PPI compared with non-smoker controls, and smokers with schizophrenia demonstrated comparable levels of PPI with control smokers [28•]. This data suggests a pro-cognitive effect of nicotine on sensory gating deficits in schizophrenia.

PPI has also been correlated with cognitive function in patients with schizophrenia. PPI and executive cognitive function using the Wisconsin Card Sorting Task (WCST) were tested in a study examining both smokers and non-smokers, and patients with schizophrenia and non-psychiatric controls [29]. In terms of PPI, non-smokers with schizophrenia displayed the most deficits, and performance on the WCST was significantly negatively correlated with greater PPI deficits in the psychiatric group, with the greatest correlations seen at the PPI $60 \mathrm{~ms}$ interval [29]. These results suggest that acute cigarette smoking may enhance the relationship between sensorimotor gating efficiency and prefrontal cognitive functioning, with smokers with schizophrenia experiencing greater PPI enhancement and thus greater performance on the WCST than non-smokers with schizophrenia. In another study, PPI was assessed at baseline, after overnight abstinence, and upon smoking reinstatement in smokers both with and without schizophrenia three times over a 3-week period. Mecamylamine, an nAChR antagonist, was administered in randomized doses of $0.0,5.0$, or $10.0 \mathrm{mg} /$ day during those 3week testing periods. At baseline, PPI was comparable in both schizophrenia and non-psychiatric controls, but was diminished in schizophrenia after overnight abstinence. 
Smoking reinstatement reversed these withdrawalinduced deficits, but was dose-dependently blocked by mecamylamine in patients, suggesting a role of nAChRs in the pathophysiology of PPI deficits [30].

\section{Working Memory}

Working memory (WM) refers to the cognitive process of manipulating and maintaining information in real time [31]. There are many different tasks used to assess WM, such as visual spatial WM (VSWM) and verbal WM, whereby patients with schizophrenia consistently display deficits [32]. Additionally, WM deficits are also found in first-degree relatives of patients with schizophrenia [33], supporting the idea of WM deficits as an endophenotype of schizophrenia.

Nicotine has been consistently demonstrated to affect WM in a number of studies. Under smoking abstinence, VSWM was significantly decreased in smokers with schizophrenia compared with non-psychiatric controls [34]. Building on this study paradigm, Sacco et al. [5] studied 25 smokers with schizophrenia and 25 nonpsychiatric smokers who were pretreated with mecamylamine, the nAChR antagonist, at doses of 0, 5.0, and $10.0 \mathrm{mg}$ /day over 3 randomized weeks [5]. VSWM was assessed at baseline, overnight abstinence, and upon smoking reinstatement. VSWM was only impaired in schizophrenia smokers after overnight abstinence, and deficits were reversed upon smoking reinstatement. Interestingly, mecamylamine effectively blocked the reinstatement of VSWM performance in schizophrenia, but not in control smokers, suggesting a central role of nAChRs in WM performance in schizophrenia [5]. These results highlight the role of nAChRs in WM in patients with schizophrenia, but more studies are needed to elucidate the effects of ad lib cigarette smoking on WM function in this population. Furthermore, specific antagonists and agonists should be used to elucidate dysfunctional receptors implicated in WM dysfunction in this patient group.

\section{Treatment Options for Nicotinic Receptor-Mediated Endophenotypes}

Varenicline

Varenicline is an $\alpha 4 \beta 2 \mathrm{nAChR}$ partial agonist at moderate concentrations, and a full $\alpha 7$ agonist at higher concentrations. Given that nicotine modulates $\alpha 4 \beta 2$ nAChRs in the mesolimbic system [35], varenicline was developed as a potential smoking cessation aid.
Randomized clinical trials of the efficacy of varenicline as a smoking cessation aid in schizophrenia have found significantly better cessation/relapse prevention rates with varenicline compared with both placebo and bupropion, another common quit-smoking drug used in this population [36, 37•]. In addition to the success of varenicline in treating smoking cessation in schizophrenia, varenicline also has been shown to have procognitive effects in this population on cognitive assessments and on some corresponding neurophysiological endophenotypes discussed above [38, 39••].

One study examined the effects of varenicline on P50 gating and cognition in both smokers and non-smokers with schizophrenia. Varenicline was administered at $0.5 \mathrm{mg} /$ day for the first week, and $1.0 \mathrm{mg}$ /day for a second week in the short-term treatment arm, and was extended to 8 weeks in the long-term arm. Results indicate that varenicline effectively reduced the P50 suppression deficit at the end of long-term treatment for nonsmokers, but not for smokers, and also improved executive function via the anti-saccadic rate. However, varenicline was not found to be effective on tasks of VSWM [39・•] (Table 1). These data suggest that the effect of chronic nicotine may normalize sensory gating, such that additional treatment with nicotinic agonists renders little to no effect. Another study demonstrated that treatment with varenicline prior to smoking abstinence in smokers with schizophrenia blocked the abstinenceinduced deficit in VSWM, but had no such effect in control smokers [40•]. Due to the limited number of studies on varenicline and WM in patients with schizophrenia, additional studies are needed to parse out the contributions of various receptor subtypes to WM deficits.

\section{DMXB-A}

3-(2,4-Dimethoxybenzylidene)-anabaseine (DMXB-A) is an $\alpha 7 \mathrm{nAChR}$ agonist that has not yet been extensively studied in regards to smoking cessation in schizophrenia. However, DMXB-A has been shown to have procognitive effects in this patient population, and also improves SPEM and sensory gating, perhaps due to stimulation of $\alpha 7 \mathrm{nAChRs}$ [41]. Administration of DMXB-A significantly enhances performance on tasks of SPEM in patients with schizophrenia, and decreases excessive hippocampal activity in response to this task in patients [42]. This evidence implicates $\alpha 7 \mathrm{nAChRs}$ in the pathophysiology of schizophrenia and highlights the need for development of an $\alpha 7$ agonist. Using these nicotinic agonists to treat these deficits may have the added benefit of acting as a smoking cessation intervention for nicotine dependence (Table 1). 


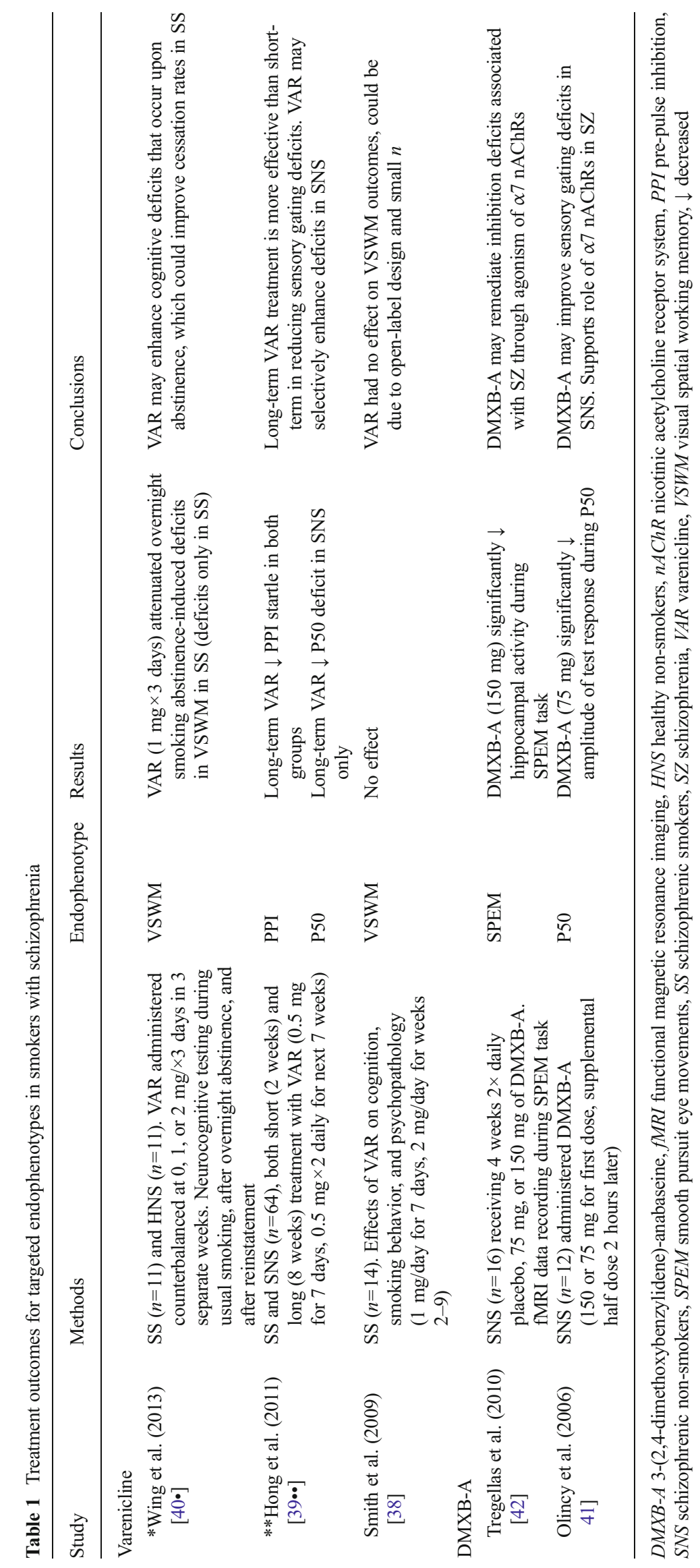


Antipsychotic Medications

Atypical antipsychotic drugs (APD) block dopamine D2 receptors in the brain, leading to increased dopamine release in the medial prefrontal cortex (mPFC) [43]. These effects are thought to reduce some of the negative and positive symptoms associated with schizophrenia, and may also play a role in smoking cessation. However, it has been suggested that patients with schizophrenia may smoke to alleviate dopamine receptor blockade by APDs. However, psychiatric symptomatology tends to improve after smoking cessation [9].

Interestingly, atypical APDs, such as clozapine, are more effective at reducing cigarette consumption in patients with schizophrenia than are typical APDs [44]. Typical APDs, such as haloperidol, may actually increase smoking rates in the schizophrenia population. In one landmark study, smokers with schizophrenia who were actively psychotic and medication-free were given 2-hour access to smoke at their leisure. In one condition, haloperidol treatment was initiated before the smoking paradigm. Participants in this group smoked significantly more cigarettes and had a higher expired carbon monoxide level compared with baseline [45].

Based on a comprehensive meta-analysis evaluating the effects of typical and atypical APDs on cognition in schizophrenia, atypical APDs were found to generally improve cognition compared with typical drugs [46]. However, some recent studies do not support the use of antipsychotic medication in alleviating cognitive dysfunction in these patients $[47$, 48]. Clearly, more specific research in this domain is required, and studies should separate smoking and non-smoking groups of patients to parse out the contribution of $\mathrm{nAChR}$ activation on cognition.

\section{Nicotine Replacement Therapies}

Nicotine replacement therapies (NRT) include nicotine gum, the nicotine patch, inhalers, nasal spray, and any other forms that deliver nicotine with the aim of replacing or reducing tobacco use [49]. Every NRT on the market is more effective than placebo at reducing smoking rates (with pooled odds of 1.58 at achieving smoking abstinence) [49].

While NRTs may be beneficial in helping non-psychiatric smokers achieve abstinence, there are mixed results regarding the effects of NRTs in the schizophrenia population. A recent meta-analysis examining the efficacy of various agents in reducing smoking found that only bupropion and varenicline were able to reduce smoking in patients with schizophrenia, and did not find an effect of NRTs [50]. In contrast to these results, one study replaced typical cigarettes with low-nicotine cigarettes, and compared their effect at reducing smoking with the nicotine patch. Both the low-nicotine cigarettes and the patch significantly reduced smoking behaviour and craving, suggesting a novel route of smoking cessation for patients with schizophrenia [51].

The self-medication hypothesis claims that patients with schizophrenia may smoke to alleviate cognitive deficits [3]. Although clinicians may be hesitant to encourage smoking cessation in patients with schizophrenia due to fears that cessation may worsen psychiatric symptomatology, evidence suggests otherwise [52]. Interestingly, a recent study found that subjective attentional benefits of cigarette smoking may not reflect objective differences in cognition, and that subjective awareness of the pro-cognitive effects of nicotine (through both patch and regular smoking) after brief deprivation was not present in patients compared with controls [53]. These results suggest that remediation of cognitive deficits is likely not the primary driving force of tobacco consumption in the schizophrenia population. The reduced efficacy of NRTs in improving cognition and reducing smoking suggests the need for more potent $\mathrm{nAChR}$ agents to ameliorating cognitive deficits with reduced $\mathrm{nAChR}$ desensitization. However, $\mathrm{nAChR}$ desensitization by smoking cessation aids may be important for achieving abstinence through mimicking the effects of nicotine at the synapse. This may account for some of the reduced efficacy of NRTs on smoking abstinence compared with more potent substrates such as varenicline, which has been shown to maximally desensitize nicotinic receptors longer than nicotine itself [54].

\section{Discussion}

Patients with schizophrenia experience the highest rates of smoking among any psychiatric diagnosis [1]. Assuming that tobacco addiction and cognitive deficits in schizophrenia share a common vulnerability, we suggest that treatment of either cognitive deficits or smoking in these patients could effectively treat the other. Ideally, treating these neurocognitive endophenotypes using nicotinic receptor (partial) agonists could help correct the underlying deficit, while providing improved observable executive functioning (including WM and sensory gating). Improved cognitive status may provide the tools patients with schizophrenia need in order to achieve better smoking cessation rates.

Among the proposed endophenotypes in schizophrenia, neurophysiological endophenotypes offer perhaps a closer representation of the underlying genotype compared with neurocognitive endophenotypes. Involvement of the CHRN A7 gene, the gene responsible for producing the $\alpha 7$ receptor subunit of nAChRs, has been linked to smoking characteristics in schizophrenia as well as to sensory gating deficiencies [55]. Abnormalities in nAChR function and sensory gating may reflect an underlying vulnerability to developing nicotine dependence in patients with schizophrenia. Other nAChR 
subtypes, including $\alpha 4 \beta 2$ receptors, have been implicated in both cognition and smoking in patients with schizophrenia due to their ability to modulate both smoking behaviors and $\mathrm{WM}[40 \bullet]$. Thus, it appears that $\mathrm{nAChR}$ dysregulation confers vulnerability to both the underlying neurophysiological endophenotype as well as resultant smoking tendencies.

Administration of nicotinic agonists and partial agonists to patients with schizophrenia who are trying to quit smoking may significantly increase cessation rates by alleviating cognitive deficits induced by tobacco withdrawal. Fittingly, several studies have already demonstrated the ability of varenicline to reverse withdrawal-induced deficits of sensory gating while simultaneously improving cognition in smokers with schizophrenia [38, 39••, 40•].

Accordingly, appropriate medication for patients with schizophrenia who are trying to quit smoking should include treatments that target both the underlying neurocognitive dysfunction as well as tobacco use disorder in these patients. Tobacco withdrawal-induced cognitive deficits may be an important reason for low cessation rates among this psychiatric population, so addressing both issues may result in increased smoking cessation success rates and improved cognitive functioning, leading to better health and quality-of-life outcomes for people with schizophrenia.

\section{Conclusion}

nAChR dysfunction may underlie some of the neurocognitive and neurophysiological deficits commonly seen in patients with schizophrenia. This receptor dysfunction could explain the high tobacco smoking prevalence in this population, and may offer a potential treatment avenue for cognitive deficits (such as SPEM, PPI, and P50) that are likely due to this aberrant receptor function. Varenicline and DMXB-A in particular may offer relief from various problems in executive functioning while reducing tobacco consumption, thereby treating both cognitive deficits and nicotine dependence through targeting the $\mathrm{nAChR}$ system.

\section{Compliance with Ethics Guidelines}

Conflict of Interest Alanna C. Bridgman, Kristen M. Mackowick, Michelle S. Goodman, Rachel A. Rabin, and Mera S. Barr declare no conflicts of interest.

Tony P. George has received consulting fees from Novartis and a grant from Pfizer has been paid to the Centre for Addiction and Mental Health, unrelated to this article.

Human and Animal Rights and Informed Consent This article does not contain any studies with human or animal subjects performed by any of the authors.

\section{References}

Papers of particular interest, published recently, have been highlighted as:

- Of importance

•- Of major importance

1. Mackowick KM, Lynch MJ, Weinberger AH, George TP. Treatment of tobacco dependence in people with mental health and addictive disorders. Curr Psychiatry Rep. 2012;14(5):478-85.

2. Lasser K, Boyd JW, Woolhandler S, Himmelstein DU, McCormick D, Bor DH. Smoking and mental illness: a population-based prevalence study. JAMA. 2000;284(20):2606-10.

3. Khantzian EJ. The self-medication hypothesis of substance use disorders: a reconsideration and recent applications. Harv Rev Psychiatry. 1997;4(5):231-44.

4. Chambers RA, Krystal JH, Self DW. A neurobiological basis for substance abuse comorbidity in schizophrenia. Biol Psychiatry. 2001;50(2):71-83.

5. Sacco KA, Termine A, Seyal A, et al. Effects of cigarette smoking on spatial working memory and attentional deficits in schizophrenia: involvement of nicotinic receptor mechanisms. Arch Gen Psychiatry. 2005;62(6):649-59.

6. Petrovsky N, Ettinger U, Quednow BB, et al. Nicotine differentially modulates antisaccade performance in healthy male non-smoking volunteers stratified for low and high accuracy. Psychopharmacology (Berl). 2012;221(1):27-38.

7. Wing VC, Bacher I, Sacco KA, George TP. Neuropsychological performance in patients with schizophrenia and controls as a function of cigarette smoking status. Psychiatry Res. 2011;188(3):3206.

8. Winterer G. Why do patients with schizophrenia smoke? Curr Opin Psychiatry. 2010;23(2):112-9.

9. Wing VC, Wass CE, Soh DW, George TP. A review of neurobiological vulnerability factors and treatment implications for comorbid tobacco dependence in schizophrenia. Ann N Y Acad Sci. 2012;1248:89-106.

10. Ferchiou A, Szoke A, Laguerre A, Meary A, Leboyer M, Schurhoff F. Exploring the relationships between tobacco smoking and schizophrenia in first-degree relatives. Psychiatry Res. 2012;200(2-3):674-8.

11. Barr RS, Culhane MA, Jubelt LE, et al. The effects of transdermal nicotine on cognition in nonsmokers with schizophrenia and nonpsychiatric controls. Neuropsychopharmacology. 2008;33(3):480-90.

12. Sherr JD, Myers C, Avila MT, Elliott A, Blaxton TA, Thaker GK. The effects of nicotine on specific eye tracking measures in schizophrenia. Biol Psychiatry. 2002;52(7):721-8.

13. Allen AJ, Griss ME, Folley BS, Hawkins KA, Pearlson GD. Endophenotypes in schizophrenia: a selective review. Schizophr Res. 2009;109(1-3):24-37.

14. Gottesman II, Gould TD. The endophenotype concept in psychiatry: etymology and strategic intentions. Am J Psychiatry. 2003;160(4):636-45.

15. Levy DL, Sereno AB, Gooding DC, O'Driscoll GA. Eye tracking dysfunction in schizophrenia: characterization and pathophysiology. Curr Top Behav Neurosci. 2010;4:311-47.

16. Tregellas JR, Tanabe JL, Miller DE, Ross RG, Olincy A, Freedman R. Neurobiology of smooth pursuit eye movement deficits in schizophrenia: an fMRI study. Am J Psychiatry. 2004;161(2): 315-21.

17. Freedman R, Hall M, Adler LE, Leonard S. Evidence in postmortem brain tissue for decreased numbers of hippocampal nicotinic receptors in schizophrenia. Biol Psychiatry. 1995;38(1):22-33. 
18. Olincy A, Johnson LL, Ross RG. Differential effects of cigarette smoking on performance of a smooth pursuit and a saccadic eye movement task in schizophrenia. Psychiatry Res. 2003;117(3):22336.

19. Potter D, Summerfelt A, Gold J, Buchanan RW. Review of clinical correlates of P50 sensory gating abnormalities in patients with schizophrenia. Schizophr Bull. 2006;32(4):692-700.

20. Chen XS, Li CB, Smith RC, Xiao ZP, Wang JJ. Differential sensory gating functions between smokers and non-smokers among drugnaive first episode schizophrenic patients. Psychiatry Res. 2011;188(3):327-33.

21. Song L, Chen X, Chen M, et al. Differences in P50 and prepulse inhibition of the startle reflex between male smokers and nonsmokers with first episode schizophrenia without medical treatment. Chin Med J (Engl). 2014;127(9):1651-5.

22. Adler LE, Hoffer LJ, Griffith J, Waldo MC, Freedman R. Normalization by nicotine of deficient auditory sensory gating in the relatives of schizophrenics. Biol Psychiatry. 1992;32(7):60716

23. Adler LE, Hoffer LD, Wiser A, Freedman R. Normalization of auditory physiology by cigarette smoking in schizophrenic patients. Am J Psychiatry. 1993;150(12):1856-61.

24. Knott VJ, Fisher DJ, Millar AM. Differential effects of nicotine on P50 amplitude, its gating, and their neural sources in low and high suppressors. Neuroscience. 2010;170(3):816-26.

25. Grillon C, Ameli R, Charney DS, Krystal J, Braff D. Startle gating deficits occur across prepulse intensities in schizophrenic patients. Biol Psychiatry. 1992;32(10):939-43.

26. Parwani A, Duncan EJ, Bartlett E, et al. Impaired prepulse inhibition of acoustic startle in schizophrenia. Biol Psychiatry. 2000;47(7):662-9.

27. Braff DL, Geyer MA, Swerdlow NR. Human studies of prepulse inhibition of startle: normal subjects, patient groups, and pharmacological studies. Psychopharmacology (Berl). 2001;156(2-3): 234-58.

28. Woznica AA, Sacco KA, George TP. Prepulse inhibition deficits in schizophrenia are modified by smoking status. Schizophr Res. 2009;112(1-3):86-90. This is an important study showing that PPI levels in smokers with schizophrenia are similar to healthy smokers, and significantly better than non-smokers with schizophrenia. These results suggest that PPI may be modulated by nicotine in schizophrenia.

29. Rabin RA, Sacco KA, George TP. Correlation of prepulse inhibition and Wisconsin Card Sorting Test in schizophrenia and controls: effects of smoking status. Schizophr Res. 2009;114(1-3):91-7.

30. George TP, Termine A, Sacco KA, et al. A preliminary study of the effects of cigarette smoking on prepulse inhibition in schizophrenia: involvement of nicotinic receptor mechanisms. Schizophr Res. 2006;87(1-3):307-15.

31. Baddeley A, Logie R, Bressi S, Della Sala S, Spinnler H. Dementia and working memory. Q J Exp Psychol A. 1986;38(4):603-18.

32. Lee J, Park S. Working memory impairments in schizophrenia: a meta-analysis. J Abnorm Psychol. 2005;114(4):599-611.

33. Myles-Worsley M, Park S. Spatial working memory deficits in schizophrenia patients and their first degree relatives from Palau, Micronesia. Am J Med Genet. 2002;114(6):609-15.

34. George TP, Vessicchio JC, Termine A, et al. Effects of smoking abstinence on visuospatial working memory function in schizophrenia. Neuropsychopharmacology. 2002;26(1):75-85.

35. Pontieri FE, Tanda G, Orzi F, Di Chiara G. Effects of nicotine on the nucleus accumbens and similarity to those of addictive drugs. Nature. 1996;382(6588):255-7

36. Williams JM, Anthenelli RM, Morris CD, et al. A randomized, double-blind, placebo-controlled study evaluating the safety and efficacy of varenicline for smoking cessation in patients with schizophrenia or schizoaffective disorder. J Clin Psychiatry. 2012;73(5):654-60.

37. Evins AE, Cather C, Pratt SA, et al. Maintenance treatment with varenicline for smoking cessation in patients with schizophrenia and bipolar disorder: a randomized clinical trial. JAMA. 2014;311(2):145-54. This maintenance trial was one of the first studies to look at the effect of varenicline on prolonged abstinence in smokers with serious mental illness after initial successful abstinence. Varenicline treatment significantly improved and lengthened abstinence without worsening psychiatric symptoms, suggesting a potential benefit of varencline for both smoking cessation and cognition

38. Smith RC, Lindenmayer JP, Davis JM, et al. Cognitive and antismoking effects of varenicline in patients with schizophrenia or schizoaffective disorder. Schizophr Res. 2009;110(1-3):149-55.

39.• Hong LE, Thaker GK, McMahon RP, et al. Effects of moderatedose treatment with varenicline on neurobiological and cognitive biomarkers in smokers and nonsmokers with schizophrenia or schizoaffective disorder. Arch Gen Psychiatry. 2011;68(12):1195206. This double-blind, parallel, randomized, placebo-controlled study examined the effects of varenicline on multiple measures of cognition in a large sample of both smokers and non-smokers with schizophrenia. Varenicline significantly reduced the P50 response in nonsmokers after long-term treatment, but had no effect on working memory, suggesting altered pathophysiology in smokers with schizophrenia compared with nonsmokers.

40. Wing VC, Wass CE, Bacher I, Rabin RA, George TP. Varenicline modulates spatial working memory deficits in smokers with schizophrenia. Schizophr Res. 2013;149(1-3):190-1. This study used a 3day paradigm of varenicline $(0.0,1.0$, or $2.0 \mathrm{mg} /$ day $x 3$ days) to evaluate its effects on cognitive performance. Varenicline was shown to attenuate abstinence-related cognitive deficits in schizophrenia, suggesting the role of $\alpha 4 \beta 2 n A C h R s$ in the pathophysiology of comorbid tobacco use in patients with schizophrenia.

41. Olincy A, Harris JG, Johnson LL, et al. Proof-of-concept trial of an alpha7 nicotinic agonist in schizophrenia. Arch Gen Psychiatry. 2006;63(6):630-8.

42. Tregellas JR, Olincy A, Johnson L, et al. Functional magnetic resonance imaging of effects of a nicotinic agonist in schizophrenia. Neuropsychopharmacology. 2010;35(4):938-42.

43. Ichikawa J, Ishii $\mathrm{H}$, Bonaccorso $\mathrm{S}$, Fowler WL, O'Laughlin IA, Meltzer HY. 5-HT(2A) and $\mathrm{D}(2)$ receptor blockade increases cortical DA release via 5-HT(1A) receptor activation: a possible mechanism of atypical antipsychotic-induced cortical dopamine release. J Neurochem. 2001;76(5):1521-31.

44. George TP, Ziedonis DM, Feingold A, et al. Nicotine transdermal patch and atypical antipsychotic medications for smoking cessation in schizophrenia. Am J Psychiatry. 2000;157(11):1835-42.

45. McEvoy JP, Freudenreich O, Levin ED, Rose JE. Haloperidol increases smoking in patients with schizophrenia. Psychopharmacology (Berl). 1995;119(1):124-6.

46. Keefe RS, Silva SG, Perkins DO, Lieberman JA. The effects of atypical antipsychotic drugs on neurocognitive impairment in schizophrenia: a review and meta-analysis. Schizophr Bull. 1999;25(2):201-22.

47. Fujimaki K, Takahashi T, Morinobu S. Association of typical versus atypical antipsychotics with symptoms and quality of life in schizophrenia. PLoS One. 2012;7(5):e37087.

48. Beninger RJ, Baker TW, Florczynski MM, Banasikowski TJ. Regional differences in the action of antipsychotic drugs: implications for cognitive effects in schizophrenic patients. Neurotox Res. 2010;18(3-4):229-43.

49. Stead LF, Perera R, Bullen C, Mant D, Lancaster T. Nicotine replacement therapy for smoking cessation. Cochrane Database Syst Rev. 2008;1, CD000146. 
50. Tsoi DT, Porwal M, Webster AC. Interventions for smoking cessation and reduction in individuals with schizophrenia. Cochrane Database Syst Rev. 2013;2, CD007253.

51. Tidey JW, Rohsenow DJ, Kaplan GB, Swift RM, Ahnallen CG. Separate and combined effects of very low nicotine cigarettes and nicotine replacement in smokers with schizophrenia and controls. Nicotine Tob Res. 2013;15(1):121-9.

52. Weinberger AH, Hitsman B, Papandonatos GD, Sacco KA, Vessicchio JC, George TP. Predictors of abstinence and changes in psychiatric symptoms in a pooled sample of smokers with schizophrenia receiving combination pharmacotherapy and behavioral therapy for smoking cessation. J Clin Psychopharmacol. 2009;29(6):601-3.
53. Hahn B, Harvey AN, Concheiro-Guisan M, Huestis MA, Holcomb HH, Gold JM. A test of the cognitive selfmedication hypothesis of tobacco smoking in schizophrenia. Biol Psychiatry. 2013;74(6):436-43.

54. Wageman CR, Marks MJ, Grady SR. Effectiveness of nicotinic agonists as desensitizers at presynaptic alpha4beta2- and alpha4alpha5beta2-nicotinic acetylcholine receptors. Nicotine Tob Res. 2014;16(3):297-305.

55. De Luca V, Wong AH, Muller DJ, Wong GW, Tyndale RF, Kennedy JL. Evidence of association between smoking and alpha7 nicotinic receptor subunit gene in schizophrenia patients. Neuropsychopharmacology. 2004;29(8):1522-6. 